\title{
A Rule Based Method to Construct The Mohr's Circle for Plane Stress
}

\author{
Swami Karunamoorthy* \\ Department of Mechanical Engineering \& Materials Science, Washington University in St. Louis Saint Louis, Missouri
}

*Corresponding author: Swami Karunamoorthy, Department of Mechanical Engineering \& Materials Science, Washington University in St. Louis Saint Louis, Missouri.
Received Date: November 15, 2019

Published Date: November 20, 2019

\section{Abstract}

Mohr's Circle is a graphical representation of the state of plane stress at a point. It is an important concept to learn for the understanding of plane stress transformation. However, it remains a challenge for both engineering as well as engineering technology students to understand the principles of Mohr's Circle. In this paper, an emphasis is given to identify the source of pedagogical difficulty and a simple rule-based method is presented as a new approach for effective teaching as well as learning. It is a viable method to understand the Mohr's Circle and its application to plane stress transformation.

\section{Introduction}

The topic on Plane Stress or Stress Transformation is introduced in a course on Mechanics of Solids or Mechanics of Materials or Strength of Materials at junior level for the undergraduate students in engineering or technology programs. The sign convention for the normal stress is well understood that tensile stress is positive and compressive stress is negative. The shear stress on an element is described as positive if the right diagonal stretches as shown in Figure 1(a).

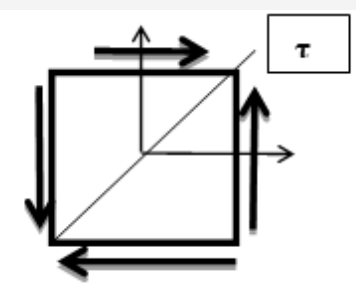

Figure 1(a): Positive shear stress on an element.

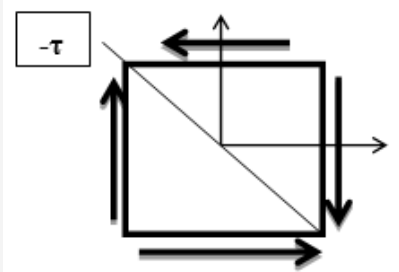

Figure 1(b): Negative shear stress on an element.
Similarly, if the left diagonal stretches as shown in Figure 1(b), the shear stress on an element is considered as negative.

However, additional sign conventions are required to draw the Mohr's Circle [1] and they are not unique. Different textbooks adopt different sign conventions for the shear on each plane (or face) of the element. Several textbooks are used as comparison for this purpose.

\section{Nomenclature}

- $\mathrm{A}_{1}$ - Top plane on the element

- $\mathrm{B}_{1}$ - Adjacent plane $\left(90^{\circ}\right.$ counterclockwise to $\left.\mathrm{A}_{1}\right)$

- $\mathrm{A}_{2}$ - Bottom plane on the element

- $\mathrm{B}_{2}$ - Adjacent plane $\left(90^{\circ}\right.$ counterclockwise to $\left.\mathrm{A}_{2}\right)$

- $\quad \mathrm{X}-\mathrm{Y}$ Reference axes

- $\mathrm{X}_{1}-\mathrm{Y}_{1}$ New position of reference axes after rotation

- $\sigma$ - Normal stress

- $\sigma_{1}, \sigma_{2}$ - Principal stresses

- $\tau$ - Shear stress

- $\quad \theta$ - Arbitrary angle of rotation

- $\theta_{\mathrm{p}}-$ Principal angle 


\section{Anomaly Among Textbooks}

In Reference [1], the author states, "To employ Mohr's circle, we must set forth additional sign conventions. A shear stress will be taken as positive on a face $\left(A_{1}\right)$ of an element in regard to Mohr's circle when it yields a clockwise moment about the center point at the element. A shear stress yielding a counterclockwise moment $\left(B_{1}\right)$ about the point will then be taken as negative." It is shown in Figure 2.

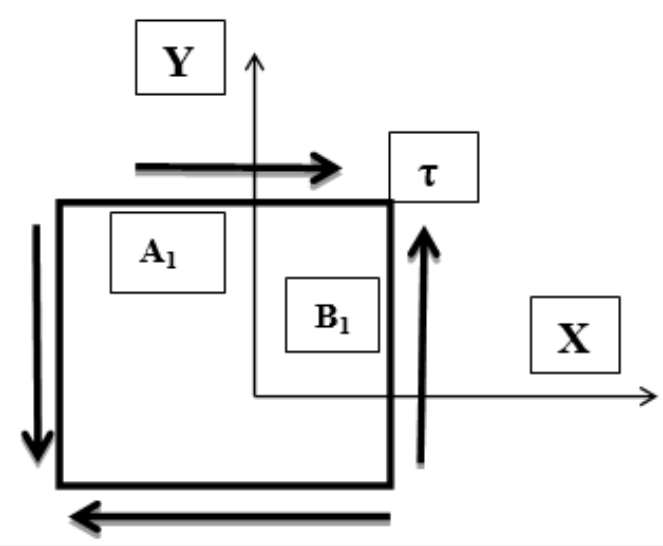

Figure 2: Sign convention for shear on Planes $A_{1}$ and $B_{1}$ [1].

This method creates a conflict with conventional method for moment based on right hand thumb rule. Based on this rule, counterclockwise moment is positive and clockwise moment is negative. For a student it results in a dilemma of Clockwise or Counterclockwise? Which one results in positive moment? However, this method for Mohr's circle [1-3] leads to correct solution while it is a pedagogical challenge for an instructor to teach something that is opposite of right-hand thumb rule. Also, it is a challenge in learning for a student.

In Reference [4], the author states, "An alternative sign convention for shear stresses is sometimes used when constructing Mohr's circle. Instead of thinking of the vertical axis as having negative shear stresses plotted upward and positive shear stresses plotted downward (which is a bit awkward), we can think of the vertical axis as having clockwise shear stresses plotted upward and counterclockwise shear stresses plotted downward." It is given in Figures 3(a) \& 3(b).

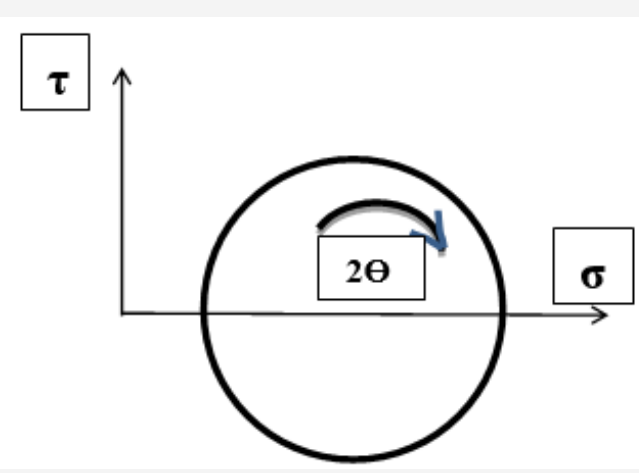

Figure 3(a): Shear axis positive upward [4].

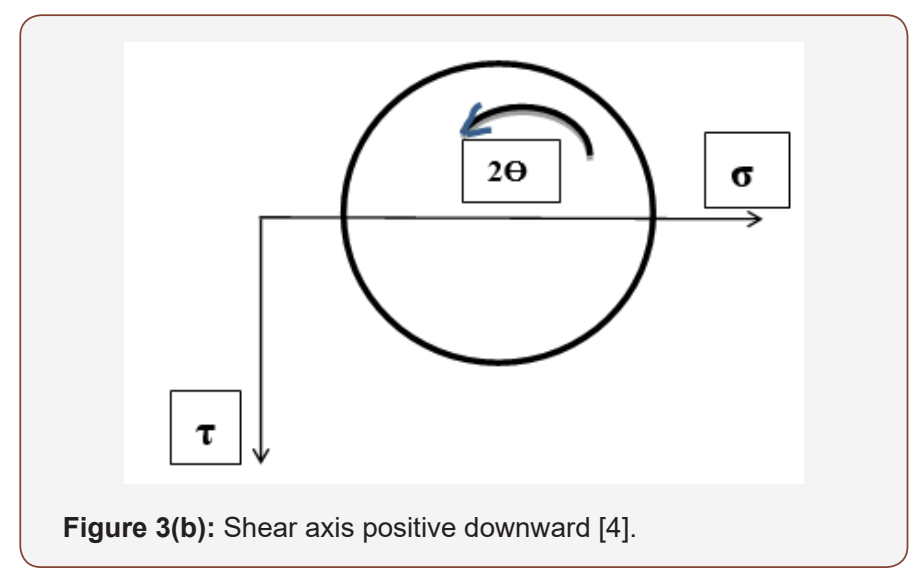

If the shear axis is positive upward, clockwise is considered as positive while if the shear axis is positive downward, counterclockwise is considered as positive. Again, it is conceptually correct; however, it is a challenge in learning to a student.

In Reference [5], the shear stress is assumed positive in the positive y \& y'-directions as shown in Figures 4(a) \& 4(b).

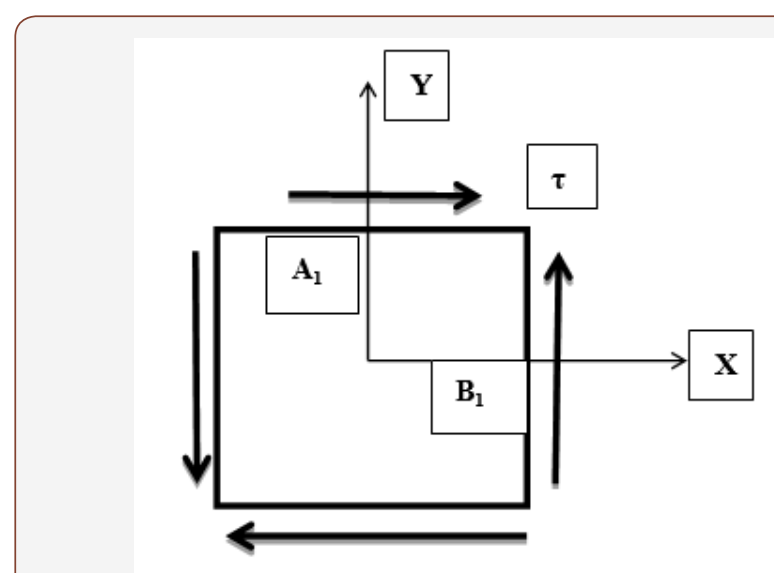

Figure 4(a): Initial position of reference axes.

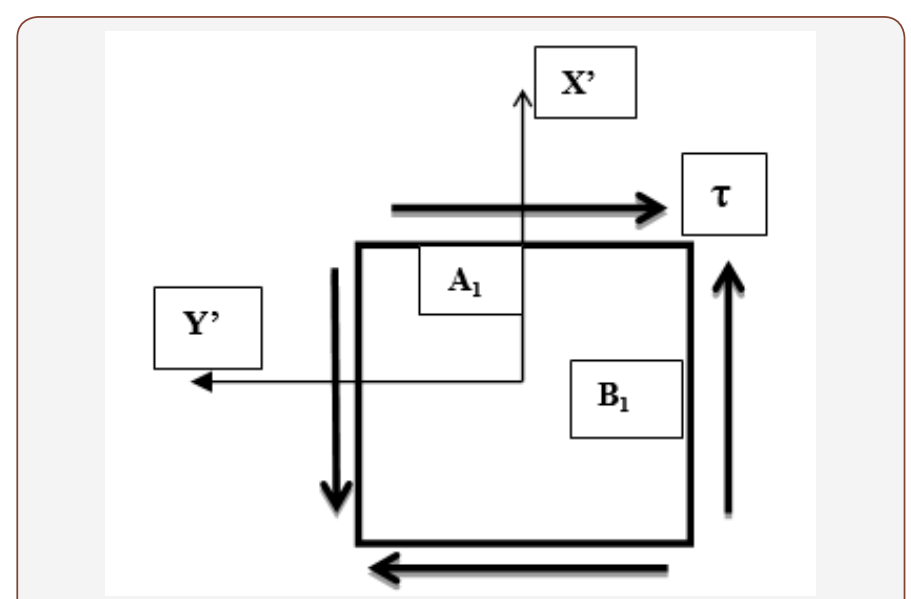

Figure 4(b): Reference axes rotated $90^{\circ}$ counterclockwise.

Based on this assumption, the shear stress on Plane-B1 is positive since it is acting along positive Y-direction as shown in Figure 4(a); while the shear stress on Plane- $\mathrm{A}_{1}$ is negative since it is acting along negative $Y^{\prime}$-direction as shown in Figure 4(b). The X-Y axes are rotated by $90^{\circ}$ counterclockwise to $X^{\prime}-Y^{\prime}$ axes to justify the 
sign of shear stress. The author states, "In other words, a rotation $\theta$ of the $X^{\prime}$ axis on the element will correspond to a rotation $2 \theta$ on the circle in the same (counterclockwise) direction." This is true for considering $\tau$-axis positive downwards in the construction of Mohr's circle. The author adds, "If instead the $\tau$-axis were constructed positive upwards, then the angle $2 \theta$ on the circle would be measured in the opposite direction to the orientation $\theta$ of the $\mathrm{X}^{\prime}$ axis." Although this approach leads to correct transformation of stresses, it is a challenge in learning and comprehension of Mohr's circle.

In Reference [6], the author does not distinguish positive or negative shear stress; however he states, "If the shear stress acting on a face of the stress element tends to rotate the stress element in a clockwise direction, then the shear stress is plotted above the $\sigma$-axis. If the shear stress tends to rotate the stress element in a counterclockwise direction, then the shear stress is plotted below the $\sigma$-axis." This method eliminates the need for whether $\tau$-axis should be positive upward or downward. Absolute values of shear stress with subscripts of ' $c w$ ' or ' $c c^{\prime}{ }^{\prime}\left(\tau_{\mathrm{cw}}, \tau_{\mathrm{ccw}}\right)$ is plotted above or below the $\sigma$-axis. Similar treatment on sign convention is encountered in Ref [7-13]. All these approaches lead to challenges in learning the concept especially to an undergraduate student in both engineering and technology programs.

\section{Rule Based Method}

Let us assume a shear is applied on top plane $\mathrm{A}_{1}$ in the positive $\mathrm{x}$-direction as shown in Figure 5(a). For force equilibrium, an equal and opposite shear is applied on bottom plane. For moment equilibrium a shear couple is applied on right plane $\left(\mathrm{B}_{1}\right)$ and left plane.

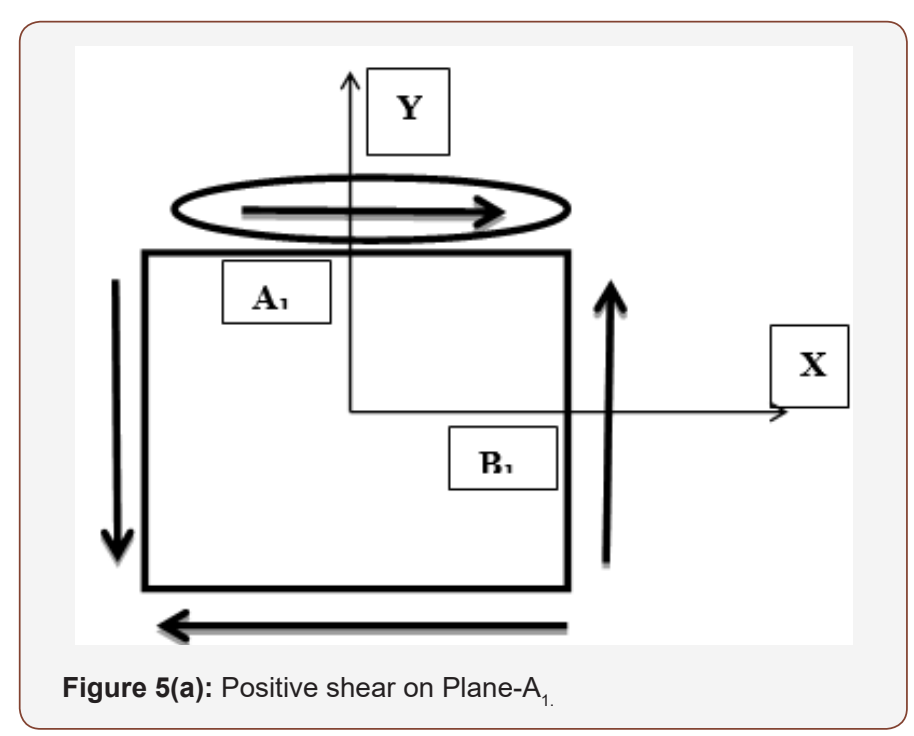

Since the applied shear on Plane $\mathrm{A}_{1}$ is in the positive $\mathrm{x}$-direction, it is intuitive to assume this shear is positive. The shear on adjacent plane $B_{1}\left(90^{\circ}\right.$ counterclockwise to $\left.A_{1}\right)$ is applied to counter the moment action of shear on plane $A_{1}$. So, the sign of shear on plane $B_{1}$ is negative of the sign of shear on plane $A_{1}$. Therefore, if the shear on plane $A_{1}$ is positive as shown in Figure 5(a), the shear on plane $B_{1}$ is negative. Similarly, if the shear on Plane $A_{1}$ is negative as shown in Figure $5(\mathrm{~b})$, the shear on Plane $B_{1}$ is positive. Although there are four planes (Top, Bottom, Right and Left) with given shear, the sign can be determined by observing the direction of shear on Top Plane alone. Therefore, a set of rules can be developed for the sign convention of shear as given below:

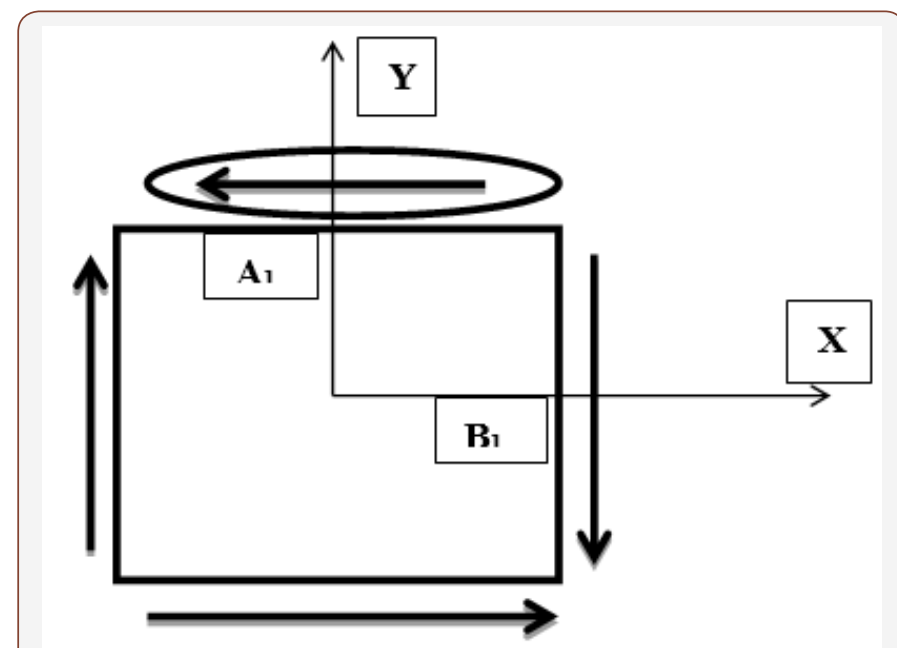

Figure 5(b): Negative shear on Plane- $\mathrm{A}_{1}$.

- Rule 1: If a shear on Plane- $\mathrm{A}_{1}$ is acting in the positive $\mathrm{X}$-direction, it is a positive shear stress

- $\quad$ Rule 2: If a shear on Plane- $\mathrm{A}_{1}$ is acting in the negative $\mathrm{X}$-direction, it is a negative shear stress

- $\quad$ Rule 3: The shear on Plane- $\mathrm{B}_{1}$ will be opposite in sign to the shear on Plane- $A_{1}$

These rules can be easily applied to construct a Mohr's circle with shear axis positive upwards. If the shear axis is positive downward, the rules are applied on planes $A_{2} \& B_{2}$ (Figure 6).

The rules on these planes would appear as:

- Rule 1: If a shear on Plane- $A_{2}$ is acting in the positive $\mathrm{X}$-direction, it is a positive shear stress

- Rule 2: If a shear on Plane- $\mathrm{A}_{2}$ is acting in the negative $\mathrm{X}$-direction, it is a negative shear stress

- $\quad$ Rule 3: The shear on Plane- $\mathrm{B}_{2}$ will be opposite in sign to the shear on Plane- $\mathrm{A}_{2}$

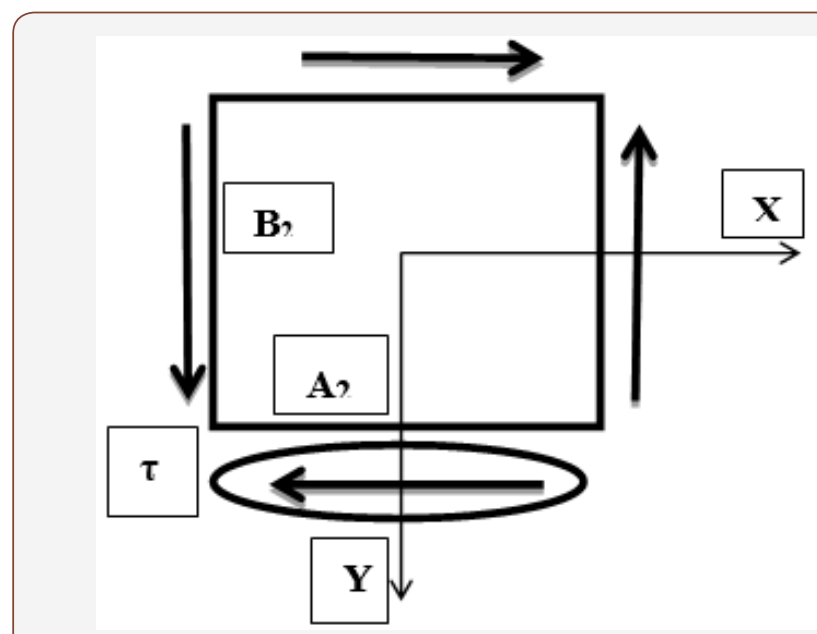

Figure 6: Shear sign convention for T-axis positive downward. 
With reference to Figure 6, the shear on Plane $A_{2}$ is negative since it is acting in the negative direction of X-axis, and hence the shear on plane $B_{2}$ is positive. To construct a Mohr's circle, a diameter is required and to draw the diameter the coordinates of two end points of the straight line is required. If the shear axis is positive upwards, apply the rules on Planes $A_{1}$ and $B_{1}$ to find the stress coordinates for Mohr's circle diameter. If the shear axis is positive downwards, apply the rules on Planes $\mathrm{A}_{2}$ and $\mathrm{B}_{2}$.

\section{Mohr's Circle}

Mohr's circle is a graphical representation of the given state of plane stress on an element. If the element is rotated through an angle $\theta$, the resulting new stresses on the element can be easily obtained from the Mohr's circle. This stress transformation is shown on the rotated element in Figure 7. If the element is rotated through an angle equivalent to the principal angle $\theta p$, the shear stress on the element vanishes while the normal stresses would be equivalent to maximum and minimum value of stresses known as principal stresses. This is shown in Figure 8 for an element rotated through an angle equivalent to principal angle.
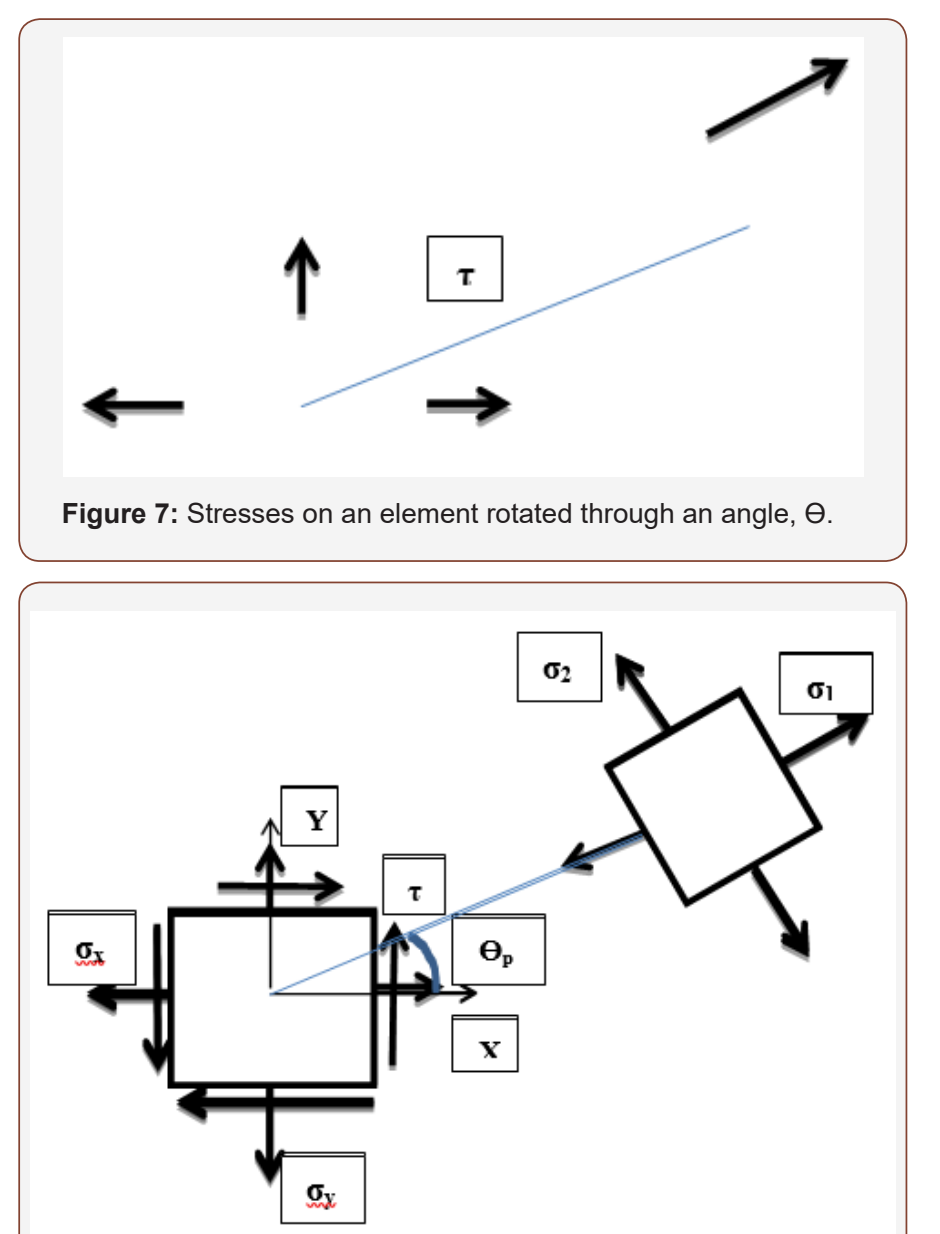

Figure 8: Stresses on an element rotated through an angle, $\Theta_{p}$.

In the construction of a Mohr's circle we are plotting the normal and shear stress values on two adjacent planes of a stress element. The graph has normal stress along the horizontal axis and shear stress along the vertical axis. In general, horizontal axis is positive to the right and vertical axis is positive upwards. If we have the stress values on two adjacent planes $\left(\mathrm{A}_{1}-\mathrm{B}_{1}\right)$ as coordinates, they can be represented on the graph as a diameter of the circle. With this diameter on the graph, a circle can be easily constructed. The rules 1,2 , \& 3 help to determine these coordinates easily without any ambiguity. If the shear axis is chosen as positive downward, the rules can be easily applied for stresses on Planes $A_{2}-B_{2}$, to determine the appropriate coordinates.

For construction of Mohr's circle, the state of stress on an element is given in Figure 9. The stress coordinates on each plane is given below:
Plane $A_{1}:(\sigma y, \tau x y)$
Plane $B_{1}:(\sigma x,-\tau x y)$.
Plane $A_{2}:(\sigma y,-\tau x y)$
Plane $B_{2}:(\sigma x, \tau x y)$.

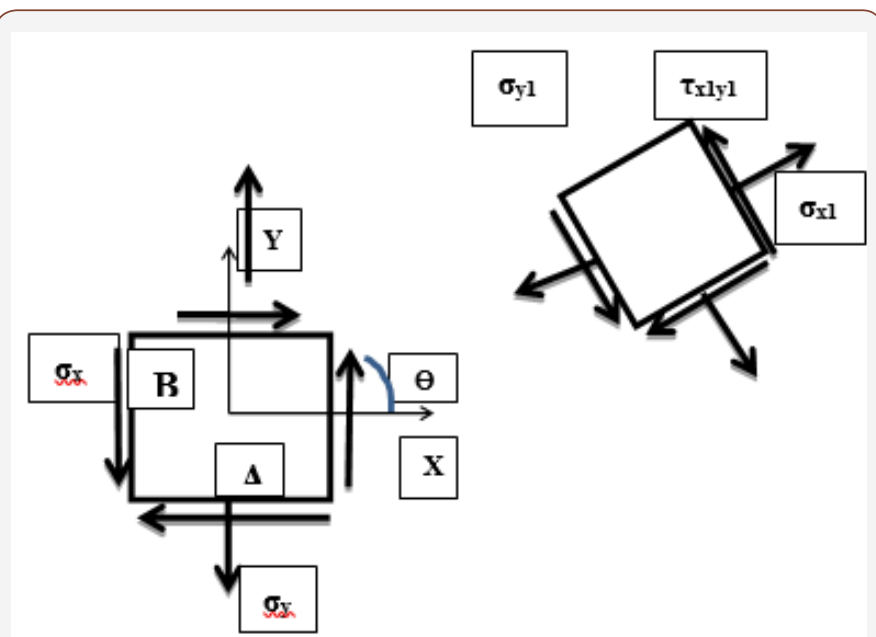

Figure 9: A general element with plane stress.

\section{Example}

To illustrate the application of rule-based method, an example problem [14] is given below in Figure 10.

$$
\sigma_{\mathrm{x}}=200 \mathrm{psi}, \sigma_{\mathrm{y}}=100 \mathrm{psi}, \tau_{\mathrm{xy}}=50 \mathrm{psi}
$$

The normal stresses are tensile stresses and hence they are positive. The shear stress on the element is positive because the shear on Plane $A_{1}$ is acting in the positive X-direction (Rule 1). The Mohr's circle is constructed with the shear axis positive upwards as well as positive downwards.

To construct a Mohr's circle with the shear axis positive upwards, the stress information on Planes $\mathrm{A}_{1}$ and $\mathrm{B}_{1}$ are:

$$
A_{1}(100,50) ; B_{1}(200,-50)
$$

To construct a Mohr's circle with the shear axis positive downwards, the stress information on Planes $\mathrm{A}_{2}$ and $\mathrm{B}_{2}$ are:

$$
A_{2}(100,-50) ; B_{2}(200,50)
$$

The Mohr's circle is presented in Figure 11. 

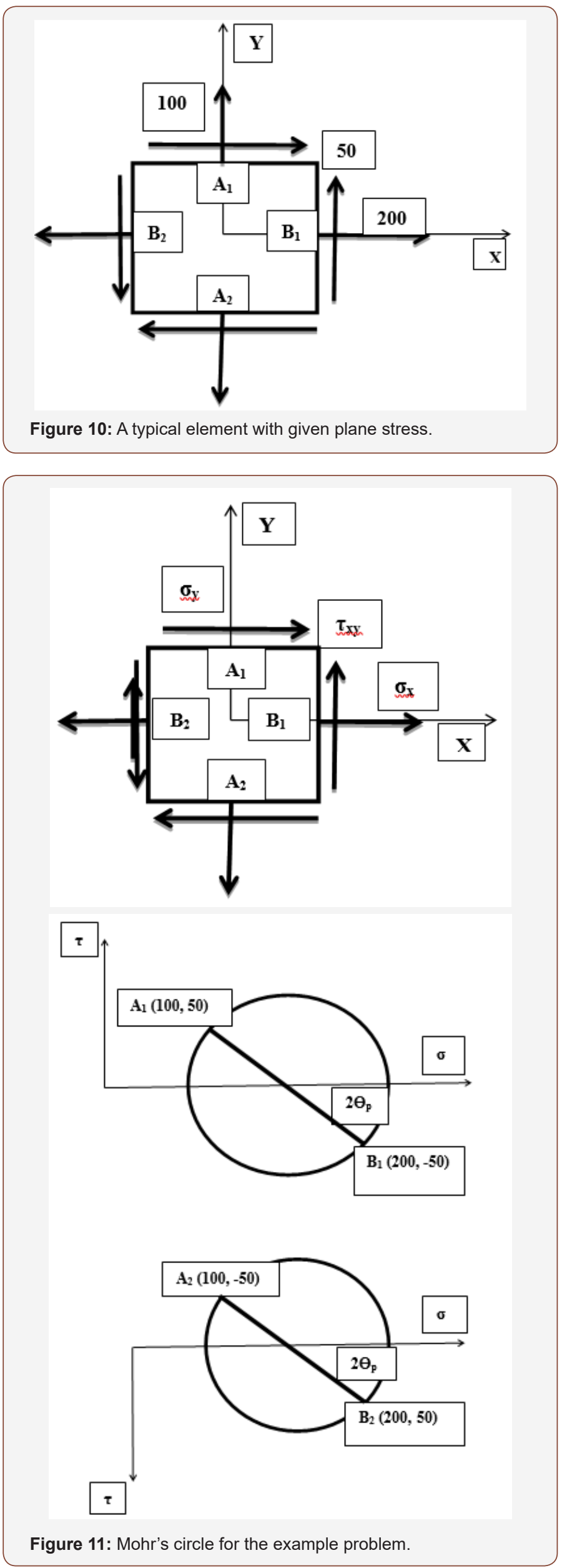

\section{Impact on Education}

The method from traditional textbooks was used for several years in teaching Mohr's circle and about fifty percent of students understood the concept. In the last few years, the rule-based method was used in the classroom resulting in remarkable improvement in understanding the concept. About ninety five percent or above students did well in the test on Mohr's circle.

\section{Conclusion}

The construction of Mohr's circle has been presented by several different methods by different authors in the literature. Although all the methods lead to correct stress transformation, it is a pedagogical challenge to present it in such a way that students can learn effectively in both engineering and technology programs. The primary challenge is to define the shear on each plane of the stress element without any ambiguity. A simple rule-based method is presented in this article to account for the shear and determine the coordinates for constructing the Mohr's circle. This method eliminates the need to know whether the shear rotate the element in clockwise or counterclockwise direction. The example problem illustrates the application of the rules and demonstrates the process for easy construction of Mohr's circle. The student performance showed significant improvement based on learning by "Rule-Based" method compared to the traditional methods from the textbooks. The rule-based method is a viable approach for effective learning of stress transformation and understand the principles of Mohr's circle.

\section{Acknowledgement}

None.

\section{Conflict of Interest}

No conflict of interest.

\section{References}

1. Shames IH (1989) Introduction to Solid Mechanics. Prentice Hall.

2. Lagace PA (2008) Mohr's Circle. Unified handout on Materials and Structures (\#M-6), MIT.

3. Ugural AC (2007) Mechanics of materials. McGraw Hill.

4. Gere JM, Goodno BJ (2012) Mechanics of Materials. Cengage Learning.

5. Hibbeler RC (1996) Mechanics of Materials. Pearson Prentice Hall.

6. Philpot TA (2016) Mechanics of Materials. John Wiley \& Sons, Inc.

7. Beer FP, Johnston ER, De Wolf JT (20140 Mechanics of Materials. McGraw Hill.

8. Davis RO, Selvadurai APS "Elasticity and geo-mechanics". Cambridge university press.

9. Holtz RD, Kovacs WD "An introduction to geotechnical engineering". Prentice Hall.

10. Parry RHG (2004) Mohr circles, stress paths and geo-technics". Taylor \& Francis.

11. Timoshenko SP, Goodier JN “Theory of Elasticity”. McGraw Hill.

12. Timoshenko SP (1983) History of Strength of Materials. Dover Publications.

13. Jaeger JC, Cook NGW, Zimmerman RW (2007) Fundamentals of rock mechanics. Wiley-Blackwell. 